\title{
Impacto do Disclosure no Risco e no Valor das Companhias Brasileiras de Capital Aberto
}

\author{
Impact of Disclosure on Risk and Value of Brazilian Public \\ Companies
}

\begin{abstract}
Valter Pereira Silva ${ }^{1}$
João Muntaser ${ }^{2}$

\section{Resumo}

O objetivo deste estudo é analisar a influência do disclosure no risco e no valor de empresas brasileiras não financeiras de capital aberto no período de 2011 a 2015. O disclosure foi medido a partir de relatórios anuais nos sites das empresas; a presença de uma sessão relacionada aos fatores de risco que podem influenciá-las e a disponibilidade de informações relacionadas ao risco, criação de valor e projeções. As variáveis de risco foram medidas pelo Beta, pelo Custo Médio Ponderado de Capital (WACC) e pela volatilidade das ações. O valor das empresas foi mensurado pelo Índice Market-to-Book, pelo valor de mercado das companhias e pelo Q de Tobin. Os resultados mostram que as empresas investigadas apresentam baixo nível de divulgação de informações no período estudado. A análise de regressão com dados em painel mostrou relação positiva e significativa entre a adesão aos níveis diferenciados de Governança Corporativa (GC) da BM\&FBovespa e o Custo Médio Ponderado de Capital. Entretanto, quando o nível de GC foi ponderado pela disponibilidade de uma sessão específica destinada aos fatores de risco, obteve-se uma relação negativa e significativa entre essa variável e o WACC. Adicionalmente, constatou-se uma relação negativa significativa entre a GC e o risco sistemático das ações (BETA). O disclosure e a GC não apresentaram relação estatisticamente significante com o valor. $\mathrm{O}$ estudo avalia a transparência no processo de comunicação entre gestores e acionistas de empresas brasileiras e mostra o papel da divulgação voluntária para os tomadores de decisão.
\end{abstract}

Palavras-Chave: Disclosure. Gestão de risco. Valor de empresas. Transparência. Empresas brasileiras.

\begin{abstract}
The objective of this study was to analyze the influence of disclosure on the risk and value of Brazilian non-financial public companies from 2011 to 2015. The disclosure was measured by annual reports on company websites; The presence of a session related to the risk factors that may
\end{abstract}

1 Faculdade de Gestão e Negócios da Universidade Federal de Uberlândia, Brasil. ORCID: https://orcid.org/0000-0002-4652-6740 . E-mail: valter1609@gmail.com

2 Faculdade de Gestão e Negócios da Universidade Federal de Uberlândia, Brasil. ORCID: https://orcid.org/0000-0002-3877-5288 .E-mail: joao.gsm@hotmail.com 
influence them and the information availability related to risk, value creation and prospections. The risk variables were measured by the Beta, the Weighted Average Cost of Capital (WACC) and the volatility of stocks. The value of the companies was measured by the Market-to-Book Index, the market value of the companies and Tobin's $Q$. The results showed that the investigated companies presented low level of information disclosure in the studied period. The regression analysis with panel data showed a significant positive relationship between the adherence to the BM\&FBovespa's Differentiated Levels of Corporate Governance (CG) and the Weighted Average Cost of Capital. However, when the CG level was weighted by the availability of a specific session for risk factors, a significant negative relationship was obtained between this variable and the WACC. Additionally, a significant negative relationship was found between the CG and the systemic risk of stocks (BETA). Disclosure and CG did not present a statistically significant relationship with the value. The study evaluates transparency in the communication process between managers and stockholders of Brazilian companies and shows the role of voluntary disclosure for the decision makers.

Keywords: Disclosure. Risk management. Value of companies. Transparency. Brazilian companies.

$$
* * *
$$

\section{Introdução}

O disclosure é o canal de comunicação entre gestores e acionistas, sendo o mesmo fundamental para a redução da assimetria informacional (Bushman \& Smith, 2003; Castro Junior, Conceição, \& Santos, 2011). Lanzana, Silveira e Famá (2006) argumentam que a divulgação de informações é uma das formas dos gestores apresentarem seu desempenho não somente para os investidores, mas também para a administração da companhia. Jensen e Meckling (1976) afirmam que o disclosure voluntário de relatórios financeiros é um mecanismo que proporciona o monitoramento dos gestores por parte de acionistas externos e também pelos credores da organização.

O trabalho de Schadewitz e Blevins (1998) chama a atenção para o papel do disclosure no mercado de capitais quando afirmam que investidores racionais evitam assumir posição acionária em companhias cuja quantidade e qualidade das informações está abaixo das expectativas. Segundo a BM\&FBovespa (2016), a adoção de boas práticas de governança corporativa confere maior credibilidade às empresas. 
Tendo em vista a importância da transparência no processo de comunicação entre gestores e acionistas e, a sua possível influência no valor das empresas e no risco assumido pelos investidores, questiona-se: Qual o impacto do disclosure no risco e no valor das companhias? O objetivo geral deste estudo consiste em analisar a influência da evidenciação de informações através do processo de disclosure no risco e no valor das companhias brasileiras de capital aberto no período de 2011 a 2015.

Este artigo baseia-se, ainda, na teoria de Disclosure. Guay e Verrecchia (2018), em estudo sobre a transparência conservadora, concluem que, muitas vezes, as empresas preferem oferecer informações de pequenos fracassos do que não tê-los pelo fato de essa ação sinalizar aos acionistas que a organização é confiável e consegue reduzir o seu risco, sendo assim recompensadas com um maior valor das ações a longo prazo. Já Leuz e Wysocki (2016) observaram que relatórios de responsabilidade ambiental corporativo não são confiáveis, com baixa qualidade das informações prestadas e, muitas vezes, as empresas preferem focar apenas nos avanços positivos. $\mathrm{Na}$ análise da obrigação regulatória sobre transparência das informações, Michelon, Pilonato e Ricceri (2015) concluíram que os resultados sobre Disclosure são realmente contraditórios e inconclusivos, justificando assim uma avaliação mais profunda sobre o tema.

Quanto ao método, este trabalho adotou as seguintes etapas: i) elaboração de um questionário semiestruturado com base no Índice de Disclosure proposto por Lanzana, Silveira e Famá (2006) para levantamento dos dados sobre transparência; ii) definição da amostra do estudo; iii) análise dos sites das empresas, em especial da parte referente às Relações com Investidores constante no site de cada empresa da amostra; iv) coleta dos dados financeiros no banco de dados Economática; v) uso de regressão com dados em painel visando medir a influência da evidenciação de informações através do processo de disclosure no risco e no valor das companhias brasileiras de capital aberto no período de 2011 a 2015. O período analisado justifica-se por ser 2011 o ano que a maioria das empresas 
do estudo começou a divulgar relatórios voluntários. Outros autores utilizam um período de 5 anos de análise (Guay \& Verrecchia, 2018), justificando a escolha de um período abrangente, possibilitando abarcar diversas particularidades dentro de um ciclo econômico.

Este estudo busca contribuir através da criação de uma medida mais abrangente de avaliação do disclosure organizacional tanto para investidores como, também, para os gestores que queiram adotar medidas de transparência em suas empresas. A criação dessa nova medida de transparência poderá contribuir com a movimentação do mercado financeiro e a inserção de novos investidores.

\section{Referencial Teórico}

\subsection{Transparência}

O Instituto Brasileiro de Governança Corporativa - IBGC (2015) define transparência como sendo o desejo de disponibilizar para as partes interessadas as informações que sejam do seu interesse e não somente as impostas por disposições de leis e regulamentos. Para Wong (2009), as organizações bem governadas buscam um nível de transparência cada vez maior, visando fornecer informações suficientes sobre o desempenho das empresas, suas perspectivas e riscos, com o intuito de facilitar decisões de investimento e reduzir a possibilidade de abusos decorrentes da assimetria de informação.

Não obstante, Dantas et al. (2005) avaliaram os benefícios da evidenciação contábil. Segundo os autores, um maior nível de disclosure beneficia os usuários com informações para o seu processo decisório e cria as condições para o desenvolvimento e a estabilidade do mercado de capitais.

No Brasil, segundo Lopes e Walker (2008), há uma estrutura insatisfatória de governança corporativa, um incentivo à manipulação dos resultados, uma presença de um mercado financeiro instável e volátil e uma fraca proteção legal aos acionistas. Rocha e Procianoy (2004) realizaram um estudo em qual concluíram que as empresas brasileiras não atribuem a 
importância devida às suas relações com investidores e analistas, deixando de aproveitarem-se de muitas vantagens que poderiam advir do seu correto uso e da transparência das informações. Sob outra perspectiva, Marques et al. (2015) analisaram a relação entre a qualidade das informações contábeis e o nível de transparência das empresas brasileiras. O estudo reforça maior capacidade explicativa dos números contábeis das empresas com maiores níveis de transparência.

No que diz respeito ao disclosure voluntário, a pesquisa de Bertomeu, Beyer e Dye (2011) desenvolveu um modelo teórico de financiamento que determina conjuntamente a estrutura de capital, a política de disclosure voluntário e o custo de capital da empresa. De acordo com esses autores, uma empresa com volatilidade baixa em seus fluxos de caixa prefere aumentar o capital pela emissão de dívidas livres de risco e adotar uma política expansiva de evidenciação de informações. Almeida e Santos (2016) analisaram se as divulgações voluntárias de informações de responsabilidade social corporativa influenciam a estrutura de capital das empresas listadas na BM\&FBovespa. Os autores constataram uma relação positiva, indicando que as empresas que disponibilizaram mais informações para o mercado conseguem captar recursos com maior facilidade.

\subsection{Transparência, Risco e Valor de Empresa: uma Revisão da Literatura}

O estudo de Lameira (2007) correlacionou governança corporativa com o risco representado pelas variáveis beta local, beta internacional, volatilidade do retorno das ações, risco não sistemático, custo médio ponderado de capital e a diferença entre o retorno da ação e o retorno da renda fixa. $\mathrm{O}$ autor encontrou uma relação negativa e significativa entre governança corporativa e risco.

Skaife, Collins e LaFond (2004) investigaram até que ponto os atributos de governança corporativa que são destinados a mitigar o risco de agência afetam o custo das empresas. Um dos resultados encontrados pelo estudo é que empresas que relatam maiores acumulações anuais e lucros 
menos transparentes têm um custo de capital mais elevado. Castro Junior, Conceição e Santos (2011) mostraram que há relação negativa e significante entre o custo de capital próprio em empresas brasileiras não financeiras e seu nível de disclosure. O estudo concluiu que maiores níveis de disclosure causam ao acionista uma percepção de risco mais baixo, e, portanto, a empresa tem como recompensa um menor custo de capital próprio.

Já o trabalho de Alves, Gonçalves e Peixoto (2014) verificou qual a relação existente entre o nível de transparência e o risco das empresas não financeiras negociadas na BM\&FBovespa no período de 2003 a 2012. Os autores sugerem que uma maior qualidade da governança corporativa pode resultar no aumento do custo do capital próprio e, em contrapartida, na redução do custo de capital de terceiros, de tal forma que o custo médio ponderado de capital da firma seja reduzido.

Entretanto, Iyengar e Lepper (2000) apontam que o excesso de informações pode resultar em uma sobrecarga na análise feita pelas pessoas, fato esse que diminui a motivação e o comprometimento no momento de escolher determinada opção ou mesmo analisar todas as informações disponíveis. Outros estudos afirmam, ainda, que o fenômeno de sobrecarga no processo de tomada de decisões pode ser agravado por contextos em que aumentos de custos estão associados à decisões equivocadas (Langer \& Rodin, 1976; Schulz, 1976; Zuckerman et al., 1978). Esse excesso de informações disponíveis possa também afetar a percepção dos investidores.

A respeito da relação entre transparência e valor de empresa, Perobelli e Ness Jr. (2000) concluíram que quando o resultado da informação divulgado é positivo, a mesma pode, sim, influenciar positivamente o preço das ações, mesmo que não seja instantaneamente. Malacrida e Yamanoto (2006) coletaram informações publicadas por 42 empresas pertencentes ao Ibovespa e constataram que empresas com maior nível de divulgação de informações apresentam menor volatilidade média no retorno de suas ações.

A pesquisa de Doná et al. (2015) analisou fatores determinantes do conteúdo divulgado no Relatório da Administração de companhias com ações 
negociadas na BM\&FBOVESPA, do setor consumo cíclico, subsetor comércio. Os autores encontraram uma relação positiva e significante entre o volume de informações contido nos Relatórios da Administração e o porte, o segmento de governança e a rentabilidade das empresas.

Michelon, Pilonato e Ricceri (2015) avaliaram a qualidade da divulgação dos relatórios de responsabilidade sustentável empresarial, um relatório voluntário. A descoberta sobre o estudo relaciona-se à uma baixa qualidade das informações divulgadas nos relatórios voluntários, tendo como consequência um certo ceticismo sobre o uso da prática de um relatório de responsabilidade sustentável empresarial como ferramenta para melhorar a responsabilização percebida. Guay e Verrecchia (2018) avaliaram o impacto da divulgação de atitudes boas e ruins das organizações em seus relatórios e como o mesmo impacta, positivamente ou negativamente, sobre o preço das ações das empresas. O estudo mostra que as empresas que comunicam atitudes negativas brandas das organizações acabam sendo vistas como de menor risco e mais confiáveis, fazendo com que os acionistas recompensem essa ação com um maior valor da ação a longo prazo.

\section{Metodologia}

\subsection{Definição da Amostra e dos Dados}

Este estudo pode ser classificado como quantitativo e descritivo, pois busca descrever as características de determinada população ou fenômeno, assim como estabelecer relações entre variáveis (Gil, 2002). No que se refere à amostra, esta foi composta pelas companhias brasileiras ativas de capital aberto e listadas na BM\&FBovespa no período de 2011 a 2015.

Esse período de análise se deve à disponibilidade dos dados nos websites das empresas. Além disso, consideraram-se somente as companhias com liquidez significativa, ou seja, aquelas que apresentaram índice de liquidez anual em bolsa superior a 0,001, com a finalidade de assegurar que as empresas da amostra apresentem um mínimo de volume de negociação de ativos, pois empresas com liquidez baixa possuem menor probabilidade de 
possuírem cotações adequadas aos valores de mercado, conforme o critério adotado por Holtz e Sarlo Neto (2014).

Por fim, foram excluídas da amostra as empresas financeiras e de fundos, em razão de suas particularidades na apuração dos resultados e de sua estrutura contábil não ser análoga às demais empresas, conforme apontam Moreira, Colauto, e Amaral (2010). Com isso, a amostra final ficou composta por 159 empresas, após os filtros de liquidez e do setor financeiro. Quanto aos dados, esta investigação utilizou dados secundários coletados no banco de dados Economática, na Comissão de Valores Mobiliários (CVM) e nos websites das empresas que compõem a amostra do estudo.

\subsection{Definição das Variáveis}

\subsubsection{Variáveis dependentes}

Para a estimação dos modelos, levando em consideração os trabalhos de Skaife, Collins e LaFond (2004), Silveira (2004), Carvalhal da Silva e Leal (2005), Lanzana, Silveira e Famá (2006), Lameira (2007), Peixoto (2012) e Alves, Gonçalves e Peixoto (2014), foram selecionadas as seguintes variáveis dependentes relacionadas ao risco e ao valor das empresas:

a) Beta (BETA): representa o risco sistemático da ação e foi mensurado através da regressão do retorno mensal do título contra o retorno mensal do Índice Bovespa. Para obtenção do beta foram utilizados os sessenta meses anteriores ao período de análise, de acordo com o modelo CAPM (Capital Asset Pricing Model);

b) Custo Médio Ponderado de Capital - CMPC (Weighted Average Cost of Capital - WACC):

$W A C C=\left\{\frac{[E B I T *(1-T)+D-1]}{(E Q U I T Y+D E B I T)} *[1+(1-\right.$ payout $\left.) * \mathrm{ROA}]\right\}+[(1-$ payout $) * \mathrm{ROA}]$

Em que: WACC = abreviatura de Weighted Average Cost of Capital, que significa Custo Médio Ponderado de Capital da empresa; EQUITY = Valor de mercado das ações da empresa; DEBT = Valor contábil da dívida da empresa; EBIT = lucros antes de juros e impostos; $\mathrm{T}$ = alíquota de imposto de renda; $\mathrm{D}$ = valor da depreciação incorrida no período; $\mathrm{I}$ $=$ valor do investimento feito pela empresa; Payout $=$ percentual do lucro destinado ao pagamento de dividendos; $\mathrm{ROA}=$ razão entre o lucro operacional e os ativos totais da empresa. 
Representa o risco da estrutura de capital da empresa, sendo uma variável proxy para o custo implícito de capital. Segundo Damodaran (2002), esse custo pode ser calculado a partir da fórmula do valor da firma, a seguir apresentada.

c) Volatilidade da ação (VOL): representa a volatilidade anual de cada ação. Esta variável foi extraída do Economática;

d) Índice preço/valor patrimonial da ação (Índice market-to-book - M/B): representa o logaritmo natural do índice preço/valor patrimonial por ação. É calculado a partir da divisão entre o preço da ação e o patrimônio líquido por ação informado no último balanço, que é calculada a partir da fórmula a seguir:

$$
M B P L=\ln \frac{P L / Q T D}{C O T}
$$

Em que: $\mathrm{MBPL}=$ Índice market-to-book; PL = patrimônio líquido da empresa; $\mathrm{QTD}=$ quantidade de ações; COT = preço de fechamento da ação.

e) Valor de Mercado (VM): representa o tamanho da empresa e foi calculado como o logaritmo natural do indicador de valor de mercado retirado da base de dados Economática;

f) Q de Tobin: representa, conforme aproximação proposta por Chung e Pruitt (1994), o valor de mercado das ações, acrescido do valor contábil das dívidas ou capital de terceiros e dividido pelo ativo total, que pode ser calculada conforme a equação abaixo:

$$
Q \text { de Tobin }=\frac{\text { VMAO }+ \text { VMAP }+ \text { DIVT }}{\text { ATIVOTOTAL }}
$$

Em que: $\mathrm{VMAO}=$ Valor de mercado das ações ordinárias; VMAP = Valor de mercado das ações preferenciais; e DIVT $=$ Valor contábil das dívidas ou capital de terceiros .

\subsubsection{Variáveis independentes}

As variáveis independentes do presente estudo foram desenvolvidas exclusivamente para o mesmo, como também foram selecionadas com base nos trabalhos de Silveira (2004), Lanzana, Silveira e Famá (2006), Peixoto (2012) e Alves, Gonçalves e Peixoto (2014): 
a) Fator Risco (FR): esta variável verificou se a empresa disponibiliza, em seu website, uma sessão específica e destinada aos fatores de risco que afetam a empresa, tais como riscos relacionados a fatores macroeconômicos, ao setor em que a companhia a ua e a empresa em si. Trata-se de uma variável binária, que assume valor 1 caso a empresa disponibilize a sessão específica em seu website e valor 0 caso não o faça;

b) Relatório Anual (RA): esta variável verificou se a empresa disponibiliza relatório anual em seu website. Trata-se de uma variável binária, que assume valor 1 caso a empresa disponibilize seu relatório anual no ano em questão e valor 0 caso não o faça. Visto que o estudo abrange o período de 2011 a 2015, foi aplicada a pontuação para cada ano analisado;

c) Risco, Criação de Valor e Projeções (RCVP): esta variável se baseou no Índice de Disclosure proposto por Lanzana, Silveira e Famá (2006), que é composto pelas informações gerais e não financeiras, pelas informações financeiras adicionais, pela análise de tendências e discussão e pela análise gerencial e pelo risco, criação de valor e projeções. Visto que o foco do presente estudo é analisar o nível de disclosure das companhias pelo risco, criação de valor e projeções, este foi utilizado no modelo econométrico. Essa medida foi construída com base na análise dos websites das empresas com foco no que foi proposto por Lanzana, Silveira e Famá (2006). Eles consideram o uso e a implementação de gestão de risco, a exposição ao risco cambial, as medidas quantitativas de criação de valor para o acionista, a compensação gerencial, as perspectivas de novos projetos, as projeções de lucro e de vendas e de crescimento. A pesquisa foi realizada com um questionário composto por 11 questões que abordaram esses temas. A pontuação das empresas nesse índice variou entre 0 e 11 pontos, sendo atribuído 1 ponto para cada resposta positiva do questionário;

d) Adesão aos níveis diferenciados de governança corporativa da BM\&FBovespa (NGC): representa a adesão das empresas aos níveis diferenciados de governança corporativa medidos pela BM\&FBovespa. Segundo a BM\&FBovespa (2016), os níveis de adesão em ordem crescente 
são: Nível 1, Nível 2 e Novo Mercado. Com isso, as empresas receberam valores de $0,1,2$ e 3, sendo 0 para o nível tradicional, que corresponde ao mercado tradicional da bolsa de valores e assim sucessivamente até 3 para empresas do nível Novo Mercado;

e) Interação entre NGC e FR ( $\left.\mathrm{NGC}^{*} \mathrm{FR}\right)$ : variável de interação que objetivou medir a relação entre a adesão aos níveis diferenciados de governança corporativa da BM\&FBovespa e a disponibilidade de uma sessão específica destinada aos fatores de risco que afetam a empresa;

f) Interação entre $\mathrm{NGC}$ e $\mathrm{RA}\left(\mathrm{NGC}^{*} \mathrm{RA}\right)$ : variável de interação que objetivou medir a relação entre a adesão aos níveis diferenciados de governança corporativa da BM\&FBovespa e a disponibilidade de relatórios anuais no website das empresas;

g) Interação entre NGC e RCVP (NGC*RCVP): variável de interação que objetivou medir a relação entre a adesão aos níveis diferenciados de governança corporativa da BM\&FBovespa e a disponibilidade das informações referentes a risco, criação de valor e projeções das empresas.

\subsubsection{Variáveis de controle}

As variáveis de controle foram selecionadas com base na possível influência que exercem sobre as variáveis dependentes de risco e valor de empresa e sobre as variáveis independentes de transparência e com base nos estudos de Lanzana, Silveira e Famá (2006), Peixoto (2012) e Alves, Gonçalves e Peixoto (2014).

a) Índice book-to-market $(\mathrm{B} / \mathrm{M})$ : índice que representa o valor patrimonial da ação sobre o seu preço. É calculado a partir do logaritmo natural do índice valor patrimonial por ação sobre o preço, que consiste na razão entre o patrimônio líquido por ação e o seu preço;

b) Valor de Empresa (VE): representa uma proxy para tamanho da empresa e foi calculado como o logaritmo natural do indicador de valor de mercado retirado da Economática; 
c) Liquidez em Bolsa (LIQ): indicador extraído da base de dados Economática que representa o volume relativo de negociações com a ação e pode ser calculado conforme a fórmula a seguir:

$$
L I Q=100 *\left(\frac{p}{p}\right) * \sqrt{\left(\frac{n}{N}\right) *\left(\frac{v}{V}\right)}
$$

Em que: LIQ $=$ Liquidez em bolsa; $p=$ número de dias em que houve pelo menos uma negociação com a ação dentro do período escolhido; $\mathrm{P}=$ número total de dias do período escolhido; $\mathrm{n}=$ número de negociações com a ação dentro do período escolhido; $\mathrm{N}=$ número de negociações com todas as ações negociadas na bolsa dentro do período escolhido; $\mathrm{v}=$ volume em dinheiro com a ação dentro do período escolhido; $\mathrm{V}=$ volume em dinheiro com todas as ações negociadas na bolsa dentro do período escolhido.

d) Crescimento de vendas (VENDAS): variável que representa a variação percentual da receita bruta total das empresas de um ano para outro;

e) Retorno sobre o Patrimônio Líquido (ROE): variável que representa a razão entre o lucro líquido do período e o valor contábil do patrimônio líquido;

f) Alavancagem financeira (AF): variável operacionalizada mediante a seguinte fórmula:

$$
A F=\frac{\frac{\frac{L L}{P L}}{L L-\text { res_fin }}}{A T}
$$

Em que: $\mathrm{LL}=$ lucro líquido; $\mathrm{PL}=$ patrimônio líquido; res_fin = resultado financeiro (receitas financeiras - despesas financeiras); AT = ativo total.

g) Setor da Empresa (SETOR): variável binária (dummy) com base no critério de classificação do banco de dados Economática, em que variáveis são classificadas como: SET1, SET2,...,SET18 (classificação das empresas em dezoito setores ao todo, com exceção do setor financeiro e de fundos).

Além das variáveis citadas, todos os modelos também foram controlados por dummies para o Ano, conforme a base de dados Economática. A Tabela 1 apresenta as variáveis utilizadas nesse estudo. 
Tabela 1. Resumo das variáveis utilizadas no estudo

\begin{tabular}{|c|c|c|c|}
\hline Nome & Variável & Natureza & Fonte/Autores /Ano \\
\hline Beta & BETA & $\begin{array}{l}\text { Variável } \\
\text { dependente }\end{array}$ & $\begin{array}{l}\text { - Economática. Lameira (2007); } \\
\text { Peixoto (2012); Alves, Gonçalves e } \\
\text { Peixoto (2014). }\end{array}$ \\
\hline $\begin{array}{l}\text { Custo Médio } \\
\text { Ponderado de } \\
\text { Capital }\end{array}$ & WACC & $\begin{array}{l}\text { Variável } \\
\text { dependente }\end{array}$ & $\begin{array}{l}\text { - Economática. Skaife, Collins e } \\
\text { LaFond (2004); Lameira (2007); } \\
\text { Peixoto (2012); Alves, Gonçalves e } \\
\text { Peixoto (2014). }\end{array}$ \\
\hline $\begin{array}{l}\text { Volatilidade da } \\
\text { ação }\end{array}$ & VOL & $\begin{array}{c}\text { Variável } \\
\text { dependente }\end{array}$ & $\begin{array}{l}\text { - Economática. Peixoto (2012); Alves, } \\
\text { Gonçalves e Peixoto (2014). }\end{array}$ \\
\hline $\begin{array}{l}\text { Índice market-to- } \\
\text { book }\end{array}$ & $\mathrm{M} / \mathrm{B}$ & $\begin{array}{l}\text { Variável } \\
\text { dependente }\end{array}$ & $\begin{array}{l}\text { - Economática. Lanzana, Silveira e } \\
\text { Famá (2006); Peixoto (2012); Alves e } \\
\text { Gonçalves e Peixoto (2014). }\end{array}$ \\
\hline Valor de mercado & VM & $\begin{array}{c}\text { Variável } \\
\text { dependente }\end{array}$ & $\begin{array}{l}\text { - Economática. Peixoto (2012); Alves, } \\
\text { Gonçalves e Peixoto (2014). }\end{array}$ \\
\hline Q de Tobin & $\begin{array}{l}\mathrm{Q} \text { de } \\
\text { Tobin }\end{array}$ & $\begin{array}{c}\text { Variável } \\
\text { dependente }\end{array}$ & $\begin{array}{l}\text { - Economática. Silveira (2004); } \\
\text { Carvalhal da Silva; Leal (2005); } \\
\text { Peixoto (2012). }\end{array}$ \\
\hline Fator Risco & FR & $\begin{array}{c}\text { Variável } \\
\text { Independente }\end{array}$ & $\begin{array}{l}\text { - Websites das empresas e CVM } \\
\text { Variável desenvolvida no estudo }\end{array}$ \\
\hline Relatório Anual & $\mathrm{RA}$ & $\begin{array}{c}\text { Variável } \\
\text { Independente }\end{array}$ & $\begin{array}{l}\text { - Websites das empresas e CVM } \\
\text { Variável desenvolvida no estudo }\end{array}$ \\
\hline $\begin{array}{l}\text { Risco, Criação de } \\
\text { Valor e Projeções }\end{array}$ & RCVP & $\begin{array}{c}\text { Variável } \\
\text { independente }\end{array}$ & $\begin{array}{l}\text { - Websites das empresas e CVM } \\
\text { Lanzana, Silveira e Famá (2006). }\end{array}$ \\
\hline $\begin{array}{l}\text { Adesão aos níveis } \\
\text { diferenciados de } \\
\text { governança da } \\
\text { BM\&FBovespa }\end{array}$ & NGC & $\begin{array}{c}\text { Variável } \\
\text { independente }\end{array}$ & $\begin{array}{l}\text { - Websites das empresas e CVM } \\
\text { Silveira (2004); Peixoto (2012); } \\
\text { Alves, Gonçalves e Peixoto (2014). }\end{array}$ \\
\hline $\begin{array}{l}\text { Índice book-to- } \\
\text { market }\end{array}$ & $\mathrm{B} / \mathrm{M}$ & $\begin{array}{l}\text { Variável de } \\
\text { controle }\end{array}$ & $\begin{array}{l}\text { - Economática. Lanzana, Silveira e } \\
\text { Famá (2006); Peixoto (2012); Alves, } \\
\text { Gonçalves e Peixoto (2014). }\end{array}$ \\
\hline Valor de Empresa & $\mathrm{VE}$ & $\begin{array}{l}\text { Variável de } \\
\text { controle }\end{array}$ & $\begin{array}{l}\text { - Economática. Lanzana, Silveira e } \\
\text { Famá (2006); Peixoto (2012); Alves, } \\
\text { Gonçalves e Peixoto (2014). }\end{array}$ \\
\hline Liquidez em bolsa & LIQ & $\begin{array}{l}\text { Variável de } \\
\text { controle }\end{array}$ & $\begin{array}{l}\text { - Economática. Lanzana, Silveira e } \\
\text { Famá (2006); Peixoto (2012); Alves, } \\
\text { Gonçalves e Peixoto (2014). }\end{array}$ \\
\hline $\begin{array}{l}\text { Crescimento de } \\
\text { vendas }\end{array}$ & VENDAS & $\begin{array}{l}\text { Variável de } \\
\text { Controle }\end{array}$ & - Economática. Peixoto (2012) \\
\hline $\begin{array}{l}\text { Retorno sobre } \\
\text { Patrimônio } \\
\text { Líquido }\end{array}$ & ROE & $\begin{array}{l}\text { Variável de } \\
\text { Controle }\end{array}$ & - Economática. Peixoto (2012) \\
\hline $\begin{array}{l}\text { Alavancagem } \\
\text { Financeira }\end{array}$ & $\overline{\mathrm{AF}}$ & $\begin{array}{l}\text { Variável de } \\
\text { Controle }\end{array}$ & $\begin{array}{l}\text { - Economática. Lanzana, Silveira e } \\
\text { Famá (2006) e Peixoto (2012) }\end{array}$ \\
\hline Setor da empresa & SETOR & $\begin{array}{l}\text { Variável de } \\
\text { controle }\end{array}$ & $\begin{array}{l}\text { - Economática. Lanzana, Silveira e } \\
\text { Famá (2006); Peixoto (2012); Alves, } \\
\text { Gonçalves e Peixoto (2014). }\end{array}$ \\
\hline
\end{tabular}




\subsection{Modelos de Regressões com Dados em Painel}

Este estudo utilizou análise de regressão com dados em painel, conforme orientações de Greene (2008) e Kennedy (2009) - Tabela 2.

Tabela 2. Modelos de regressões com dados em painel

\begin{tabular}{|c|c|c|c|c|}
\hline $\begin{array}{l}\text { Descrição do } \\
\text { Modelo }\end{array}$ & $\begin{array}{l}\text { Mod } \\
\text { elos }\end{array}$ & $\begin{array}{c}\text { Variável } \\
\text { Dependente }\end{array}$ & $\begin{array}{c}\text { Variáveis } \\
\text { Independentes }\end{array}$ & Variáveis de Controle \\
\hline $\begin{array}{c}\text { Modelo de } \\
\text { Efeitos Fixos }\end{array}$ & 1 & BETA & $\begin{array}{l}\mathrm{FR}, \mathrm{NGC} \\
\mathrm{NGC} * \mathrm{FR}\end{array}$ & $\begin{array}{l}\text { WACC, VOL, B/M, VE, } \\
\text { LIQ, ANO, SETOR }\end{array}$ \\
\hline $\begin{array}{c}\text { Modelo de } \\
\text { Efeitos Fixos }\end{array}$ & 2 & BETA & $\begin{array}{l}\text { RA, NGC, } \\
\text { NGC*RA }\end{array}$ & $\begin{array}{l}\text { WACC, VOL, B/M, VE, } \\
\text { LIQ, ANO, SETOR }\end{array}$ \\
\hline $\begin{array}{c}\text { Modelo de } \\
\text { Efeitos Fixos }\end{array}$ & 3 & BETA & $\begin{array}{l}\text { RCVP, NGC, } \\
\text { NGC*RCVP }\end{array}$ & $\begin{array}{l}\text { WACC, VOL, B/M, VE, } \\
\text { LIQ, ANO, SETOR }\end{array}$ \\
\hline $\begin{array}{c}\text { Modelo de } \\
\text { Efeitos Fixos }\end{array}$ & 4 & WACC & $\begin{array}{l}\text { FR, NGC, } \\
\text { NGC*FR }\end{array}$ & $\begin{array}{l}\text { BETA, VOL, B/M, VE, } \\
\text { LIQ, ANO, SETOR }\end{array}$ \\
\hline $\begin{array}{c}\text { Modelo de } \\
\text { Efeitos Fixos }\end{array}$ & 5 & WACC & $\begin{array}{l}\text { RA, NGC, } \\
\mathrm{NGC}^{*} \mathrm{RA}\end{array}$ & $\begin{array}{l}\text { BETA, VOL, B/M, VE, } \\
\text { LIQ, ANO, SETOR }\end{array}$ \\
\hline $\begin{array}{l}\text { Modelo de } \\
\text { Efeitos Fixos }\end{array}$ & 6 & WACC & $\begin{array}{l}\text { RCVP, NGC, } \\
\text { NGC*RCVP }\end{array}$ & $\begin{array}{l}\text { BETA, VOL, B/M, VE, } \\
\text { LIQ, ANO, SETOR }\end{array}$ \\
\hline $\begin{array}{c}\text { Modelo de } \\
\text { Efeitos Fixos }\end{array}$ & 7 & VOL & $\begin{array}{l}\text { FR, NGC, } \\
\text { NGC*FR }\end{array}$ & $\begin{array}{l}\text { WACC, BETA, B/M, VE, } \\
\text { LIQ, ANO, SETOR }\end{array}$ \\
\hline $\begin{array}{l}\text { Modelo de } \\
\text { Efeitos Fixos }\end{array}$ & 8 & VOL & $\begin{array}{l}\text { RA, NGC, } \\
\text { NGC*RA }\end{array}$ & $\begin{array}{l}\text { WACC, BETA, B/M, VE, } \\
\text { LIQ, ANO, SETOR }\end{array}$ \\
\hline $\begin{array}{c}\text { Modelo de } \\
\text { Efeitos Fixos }\end{array}$ & 9 & VOL & $\begin{array}{l}\text { RCVP, NGC, } \\
\text { NGC*RCVP }\end{array}$ & $\begin{array}{l}\text { WACC, BETA, B/M, VE, } \\
\text { LIQ, ANO, SETOR }\end{array}$ \\
\hline $\begin{array}{c}\text { Modelo de } \\
\text { Efeitos Fixos }\end{array}$ & 10 & $\mathrm{M} / \mathrm{B}$ & $\begin{array}{l}\text { FR, NGC, } \\
\text { NGC*FR }\end{array}$ & $\begin{array}{l}\text { AF, VENDAS, ROE, } \\
\text { LIQ, ANO, SETOR }\end{array}$ \\
\hline $\begin{array}{c}\text { Modelo de } \\
\text { Efeitos Fixos }\end{array}$ & 11 & $\mathrm{M} / \mathrm{B}$ & $\begin{array}{l}\text { RA, NGC, } \\
\text { NGC*RA }\end{array}$ & $\begin{array}{l}\text { AF, VENDAS, ROE, } \\
\text { LIQ, ANO, SETOR }\end{array}$ \\
\hline $\begin{array}{c}\text { Modelo de } \\
\text { Efeitos Fixos }\end{array}$ & 12 & $\mathrm{M} / \mathrm{B}$ & $\begin{array}{l}\text { RCVP, NGC, } \\
\text { NGC*RCVP }\end{array}$ & $\begin{array}{l}\text { AF, VENDAS, ROE, } \\
\text { LIQ, ANO, SETOR }\end{array}$ \\
\hline $\begin{array}{l}\text { Modelo de } \\
\text { Efeitos Fixos }\end{array}$ & 13 & VM & $\begin{array}{l}\text { FR, NGC, } \\
\text { NGC*FR }\end{array}$ & $\begin{array}{l}\text { AF, VENDAS, ROE, } \\
\text { LIQ, ANO, SETOR }\end{array}$ \\
\hline $\begin{array}{c}\text { Modelo de } \\
\text { Efeitos Fixos }\end{array}$ & 14 & VM & $\begin{array}{l}\text { RA, NGC, } \\
\text { NGC*RA }\end{array}$ & $\begin{array}{l}\text { AF, VENDAS, ROE, } \\
\text { LIQ, ANO, SETOR }\end{array}$ \\
\hline $\begin{array}{c}\text { Modelo de } \\
\text { Efeitos Fixos }\end{array}$ & 15 & $\mathrm{VM}$ & $\begin{array}{l}\text { RCVP, NGC, } \\
\text { NGC*RCVP }\end{array}$ & $\begin{array}{l}\text { AF, VENDAS, ROE, } \\
\text { LIQ, ANO, SETOR }\end{array}$ \\
\hline $\begin{array}{l}\text { Modelo de } \\
\text { Efeitos } \\
\text { Aleatórios }\end{array}$ & 16 & Q de Tobin & $\begin{array}{l}\text { FR, NGC, } \\
\text { NGC*FR }\end{array}$ & $\begin{array}{l}\text { AF, VENDAS, ROE, } \\
\text { LIQ, ANO, SETOR }\end{array}$ \\
\hline $\begin{array}{l}\text { Modelo de } \\
\text { Efeitos } \\
\text { Aleatórios }\end{array}$ & 17 & Q de Tobin & $\begin{array}{l}\text { RA, NGC, } \\
\text { NGC*RA }\end{array}$ & $\begin{array}{l}\text { AF, VENDAS, ROE, } \\
\text { LIQ, ANO, SETOR }\end{array}$ \\
\hline $\begin{array}{l}\text { Modelo de } \\
\text { Efeitos } \\
\text { Aleatórios }\end{array}$ & 18 & $\mathrm{Q}$ de Tobin & $\begin{array}{l}\text { RCVP, NGC, } \\
\text { NGC*RCVP }\end{array}$ & $\begin{array}{l}\text { AF, VENDAS, ROE, } \\
\text { LIQ, ANO, SETOR }\end{array}$ \\
\hline
\end{tabular}

Inicialmente, realizou-se o teste de Lagrange Multiplier (LM) para definir entre os modelos Pooled ou Painel; a seguir, procedeu-se com o teste 
de Hausman para definição entre a utilização de modelos de efeitos fixos ou aleatórios. Ademais, adotou-se os testes de heterocedasticidade de Baum (2001) e o teste de autocorrelação de Wooldridge (2002), utilizando o comando robust no Stata 13.

Dessa forma, obteve-se 18 (dezoito) modelos econométricos para análise das regressões que relacionam o disclosure e o risco e o disclosure e o valor de empresa, conforme exposto no Tabela 2. Para cada uma das 6 (seis) variáveis dependentes, havia 3 (três) especificações de modelos devido às variáveis explicativas de disclosure que variavam em cada modelo (Fator de Risco - FR; Relatório de Administração - RA; e RCVP ).

\section{Análise de Resultados}

A discussão e análise dos resultados da pesquisa têm início com a exposição da Tabela 3, que apresenta as estatísticas descritivas do estudo.

Tabela 3. Estatística descritiva da pesquisa

\begin{tabular}{|c|c|c|c|c|c|}
\hline Variável & Observações & Média & $\begin{array}{l}\text { Desvio } \\
\text { Padrão }\end{array}$ & Mínimo & Máximo \\
\hline BETA & 710 & 0,7929437 & 0,4478775 & 0,01 & 1,87 \\
\hline WACC & 806 & 1.783 .597 & $5,33456-10$ & $0,824879$. & 122579 \\
\hline VOL & 851 & 3.496 .214 & 1.620 .354 & 17,26 & 72,24 \\
\hline $\mathrm{M} / \mathrm{B}$ & 892 & 1.684 .497 & $1,442854 \quad .1$ & 3392434 . & 912459 \\
\hline VM & 892 & $5,35 \mathrm{e}+09$ & $5,88 \mathrm{e}+09$ & $92 \mathrm{e}+08 \quad 1$ & $7,60 \mathrm{E}+11$ \\
\hline Q de Tobin & 892 & 1.366 .775 & $12,69552-10$ & $0,21819 \quad 3$ & 13,941 \\
\hline FR & 925 & 0,4108108 & 0,4922471 & 0 & 1 \\
\hline $\mathrm{RA}$ & 925 & 0,2237838 & 0,4170043 & 0 & 1 \\
\hline RCVP & 925 & 0,8216216 & 1.791 .505 & 0 & 9 \\
\hline NGC & 925 & 1.994 .595 & 1.285 .523 & 0 & 3 \\
\hline $\mathrm{B} / \mathrm{M}$ & 806 & 0,9690553 & 1,319337 .0 & 0127283 & 479335 \\
\hline VM & 892 & $5,35 \mathrm{e}+09$ & $5,88 \mathrm{e}+091$ & $92 \mathrm{e}+08 \quad 1$ & $7,60 \mathrm{E}+11$ \\
\hline LIQ & 925 & 0,2580908 & 0,332492 & 0,001 & 1,019 \\
\hline VENDAS & 843 & 370.963 & $\begin{array}{ll}18,31358 & -29\end{array}$ & $0,47021 \quad 41$ & 0,54621 \\
\hline ROE & 829 & 6.907 .831 & -1.408 .986 & $23.414 \quad 26$ & 0,91738 \\
\hline $\mathrm{AF}$ & 895 & 1.718 .022 & $\begin{array}{ll}1,398075 \quad .2\end{array}$ & 1665654 . & 815101 \\
\hline
\end{tabular}

Observa-se na Tabela 3 que as empresas consideradas na amostra apresentaram baixo nível de divulgação de informações. Como exemplo disso, tem-se o fato de que a média de pontuação atingida pelas companhias estudadas foi de 0,41 pontos, variando entre 0 e 1 , sendo 0 para empresas 
que não têm uma sessão específica destinada a informações referentes aos riscos que podem influenciá-las e 1 ponto para empresas que mantêm tal seção. Outro resultado que confirma o baixo nível de disclosure das companhias é a pontuação média alcançada pelas empresas na disponibilização do relatório anual, que resultou em 0,22 pontos, variando, também, entre 0 para empresas que não disponibilizam tal informação e 1 ponto para companhias que o fazem.

Informações com relação ao Risco, Criação e Valor e Projeções foram analisadas por Lanzana, Silveira e Famá (2006), com base no Índice de Disclosure proposto pelos autores. Eles analisaram o uso e a implementação dessas informações através de um questionário composto por 11 questões que abordaram esses temas. A pontuação máxima alcançada pelas empresas foi de 9 pontos (variando entre 0 e 11); entretanto, a média dessa pontuação foi de 0,82, valor esse que confirma o baixo nível de disclosure das companhias analisadas. Esses resultados vão ao encontro dos obtidos por Rocha e Procianoy (2004) e Lopes e Walker (2008), os quais atestaram uma despreocupação com o disclosure no Brasil, resultando em um baixo nível de informação voluntária de suas organizações listadas na bolsa de valores.

O estudo segue com a exposição da Tabela 4, que apresenta os modelos de regressões de dados em painel que mostram as variáveis relacionadas ao disclosure com as variáveis relacionadas ao risco, conforme foi detalhado na metodologia.

Conforme o modelo 1 , percebe-se uma relação positiva e estatisticamente significante ao nível de 10\% entre a adesão aos níveis diferenciados de GC da BM\&FBovespa (NGC) e o Custo Médio Ponderado de Capital (WACC). Esses resultados contrariam os encontrados no estudo de Alves, Gonçalves e Peixoto (2014), que concluiu que empresas em um nível maior de GC apresentam um WACC mais baixo. Além disso, tal resultado contraria os encontrados nos estudos de Castro Junior, Conceição e Santos (2011), Almeida e Santos (2016), pois esperava-se que, com um maior nível 
de transparência e qualidade de informações, houvesse captação de recursos menos onerosa; entretanto, não é isso o que os resultados apontam.

Tabela 4. Modelos de regressões que relacionam disclosure e risco

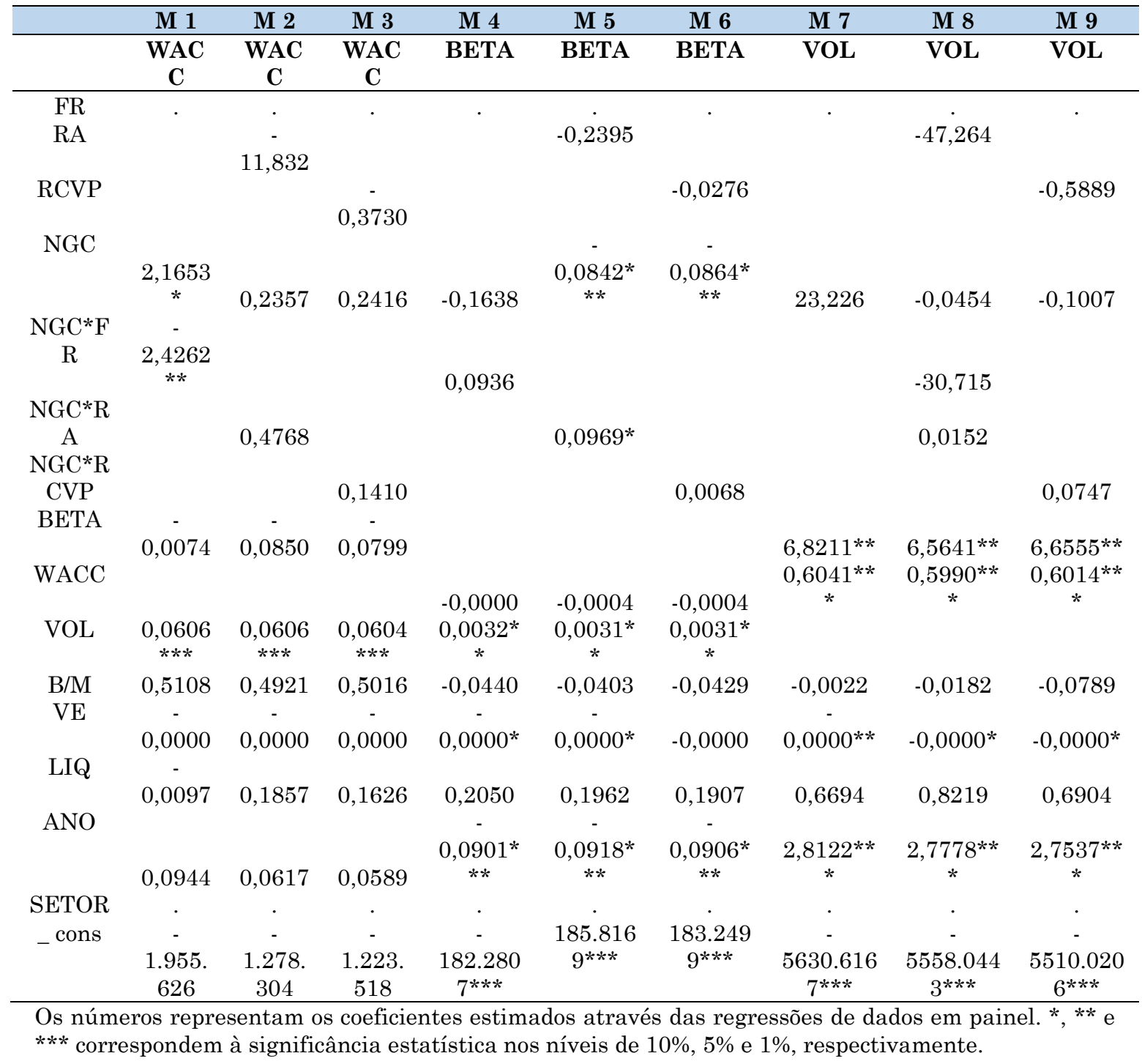

Vale ressaltar que, quando o NGC foi ponderado com a disponibilidade de uma sessão específica para os fatores de risco que afetam a empresa $(\mathrm{NGC} * \mathrm{FR})$, conforme o modelo 1, obteve-se uma relação negativa e estatisticamente significante ao nível de 5\%, entre essa variável e o WACC, resultado este que está alinhado com o obtido em outros trabalhos (Almeida \& Santos, 2016; Alves, Gonçalves, \& Peixoto, 2014; Castro Júnior, Conceição, \& Santos, 2011). Com isso, sugere-se que, para a empresa colher os frutos de um custo de capital reduzido, não é suficiente encontrar-se em 
um nível de GC diferenciado; é preciso que sejam, também, disponibilizadas informações não obrigatórias, tais como os riscos que podem afetar a companhia.

Adicionalmente, conforme os modelos 5 e 6, constatou-se uma relação negativa e estatisticamente significante ao nível de $1 \%$ entre a variável NGC e o risco sistemático das ações (BETA). Esses resultados estão em consonância com os encontrados em Lameira (2007) e Alves, Gonçalves e Peixoto (2012) e indicam que aumentos significativos no nível de GC provocam um resultado inversamente proporcional no risco das empresas. Entretanto, quando o NGC é ponderado pela disponibilidade do Relatório Anual, ocorre a inversão do sinal, fato esse que sugere que empresas que disponibilizam uma informação voluntária e que, ao mesmo tempo estão em níveis diferenciados de GC, aumentam o seu risco.

Visto que a literatura não tem uma resposta definitiva para esse resultado, supõe-se que o mesmo esteja relacionado à racionalidade limitada dos acionistas. Diante do fato que os mesmos recebem, diariamente, um grande fluxo de informações e, muitas vezes, não conseguem lidar com as mesmas. Assim, supõe-se que possa ocorrer uma sobrecarga no processo de tomada de decisões que pode ser agravado por aumentos nos custos associados a decisões equivocadas (Iyengar \& Lepper, 2000; Langer \& Rodin, 1976; Schulz, 1976; Zuckerman et al., 1978).

Ademais, observou-se durante a coleta de dados que a disponibilização dessa informação é inconstante para a maioria das empresas, ou seja, em alguns anos isso é feito e em outros não. Sugere-se que as companhias prefiram liberar esse relatório quando o mercado verifica um risco maior com relação à empresa, com o intuito de acalmar os ânimos dos acionistas.

Posteriormente, tem-se a Tabela 5, que apresenta os modelos de regressões de dados em painel que relacionam as variáveis relacionadas ao disclosure com as variáveis relacionadas ao valor das empresas, conforme detalhamento feito na metodologia. 
Tabela 5. Modelos de regressões que relacionam disclosure e valor de empresa

\begin{tabular}{|c|c|c|c|c|c|c|c|c|c|}
\hline & M 10 & M 11 & M 12 & M 13 & M 14 & M 15 & M 16 & M 17 & M 18 \\
\hline & $\mathbf{M} / \mathbf{B}$ & $\mathbf{M} / \mathbf{B}$ & $\mathbf{M} / \mathbf{B}$ & VM & VM & VM & $\begin{array}{c}\text { Q de } \\
\text { Tobin }\end{array}$ & $\begin{array}{c}\text { Q de } \\
\text { Tobin }\end{array}$ & $\begin{array}{c}\text { Q de } \\
\text { Tobin }\end{array}$ \\
\hline FR & . & . & . & . & & . & 22,664 & . & \\
\hline RA & & $-0,0387$ & & & $1,29 \mathrm{E}+12$ & & & $\begin{array}{c}- \\
0,3027\end{array}$ & \\
\hline RCVP & & & 0,0271 & & & $3,88 \mathrm{E}+11$ & & & 0,2196 \\
\hline NGC & 0,3183 & $-0,1044$ & $-0,1057$ & $2,58 \mathrm{E}+12$ & $3,45 \mathrm{E}+11$ & $3,33 E+11$ & 0,6425 & 0,3742 & 0,5063 \\
\hline NGC $* F$ & & & & - & & & - & & \\
\hline $\mathrm{R}$ & $-0,5296$ & & & $2,79 \mathrm{E}+12$ & & & 0,4328 & & \\
\hline NGC*R & & & & & - & & & & \\
\hline A & & 0,1090 & & & $1,33 \mathrm{E}+11$ & & & 0,7662 & \\
\hline NGC*R & & & & & & - & & & - \\
\hline CVP & & & 0,0163 & & & $1,05 \mathrm{E}+11$ & & & 0,0076 \\
\hline \multirow[t]{2}{*}{$\mathrm{AF}$} & $0,2964 * *$ & $0,3091 * *$ & $0,3061 * *$ & - & - & - & - & - & - \\
\hline & $*$ & $*$ & $*$ & $8,48 E+11$ & $7,77 \mathrm{E}+11$ & $8,13 E+11$ & 0,3417 & 0,4135 & 0,3953 \\
\hline VENDA & & & & $3,870 \mathrm{e}+0$ & $3,698 \mathrm{e}+0$ & $3,810 \mathrm{e}+0$ & 0,0914 & 0,0907 & 0,0919 \\
\hline $\mathrm{S}$ & $0,0055^{* *}$ & $0,0051 * *$ & $0,0054 * *$ & $7 * * *$ & $7 * * *$ & $7 * * *$ & $* *$ & $* *$ & $* *$ \\
\hline \multirow[t]{2}{*}{ ROE } & & & & - & - & - & 0.1199 & 0.1158 & 0.1185 \\
\hline & $0,0135^{* *}$ & $0,0128 * *$ & $0,0126 * *$ & $1,50 \mathrm{E}+10$ & $1,84 \mathrm{E}+10$ & $1,82 \mathrm{E}+10$ & $* *$ & ** & $* *$ \\
\hline \multirow[t]{2}{*}{ LIQ } & $0,7750 * *$ & $0,8987 * *$ & $0,8883 * *$ & $6,115 \mathrm{e}+0$ & $6,749 \mathrm{e}+0$ & $6,710 \mathrm{e}+0$ & & & \\
\hline & $*$ & $*$ & $*$ & $9 * * *$ & $9 * * *$ & $9 * * *$ & 0,8767 & 0,5075 & 0,5451 \\
\hline \multirow{3}{*}{ ANO } & - & - & - & & & & & & \\
\hline & $0,1565 * *$ & $0,1598 * *$ & $0,1561 * *$ & - & - & - & & & \\
\hline & $*$ & $*$ & $*$ & $2,83 \mathrm{E}+10$ & $3,02 \mathrm{E}+10$ & $6,86 \mathrm{E}+09$ & 0,1667 & 0,1630 & 0,1881 \\
\hline \multirow{3}{*}{$\begin{array}{c}\text { SETOR } \\
\text { _ cons }\end{array}$} & . & . & . & . & . & . & 0,0098 & 0,0127 & 0,0121 \\
\hline & & & & & & & - & - & - \\
\hline & $\begin{array}{c}315.7056 \\
* * *\end{array}$ & $\begin{array}{c}322.6594 \\
* * *\end{array}$ & $\begin{array}{c}315.3123 \\
* * *\end{array}$ & $6,19 \mathrm{E}+13$ & $6.73 E+13$ & $2.05 E+13$ & $\begin{array}{c}3.238 \\
889\end{array}$ & $\begin{array}{c}3.154 . \\
046\end{array}$ & $\begin{array}{c}3.660 . \\
635\end{array}$ \\
\hline
\end{tabular}

Conforme os modelos da tabela 3, percebe-se que as variáveis relacionadas ao disclosure e à $\mathrm{GC}$ não apresentaram relação estatisticamente significante com as variáveis relacionadas ao valor. Tais resultados diferem dos encontrados nos trabalhos de Perobelli e Ness Jr. (2000) e Doná et al. (2015), que encontraram uma relação positiva e estatisticamente significante entre a transparência e o valor.

Entretanto, um importante resultado, encontrado no presente estudo, consiste em até que ponto a informação é necessária para o investidor e quando a mesma extrapola seus limites de compreensão. Observando-se a transparência em níveis, o fator de risco é o primeiro deles, no qual é uma informação simples, rápida, curta e disponível no site da organização. $O$ segundo é a disponibilidade do relatório anual, uma informação mais trabalhosa de se elaborar e organizar, sendo mais difícil para a análise do 
investidor. O terceiro nível é a qualidade dessas informações, mensurado nesse trabalho pelo RCVP.

O resultado alcançado sugere que os níveis de informação impactam o risco da empresa, e não o valor da mesma, conforme significância estatística para as variáveis de interesse expostos na tabela 2. Outra informação interessante é o fato de que o nível dessa informação também impacta a percepção do risco, tanto assim que, para o mais elevado RCVP, não foi encontrado significância com nenhuma das regressões em que o mesmo foi testado, sugerindo que existe um limite para captação e o processamento de informações pelos acionistas, atingindo um nível em que a mesma se torna indiferente para o esse investidor, tornando-se um esforço desperdiçado pela da organização.

\section{Conclusões}

O presente estudo teve como objetivo principal analisar a influência do disclosure no risco e no valor das empresas brasileiras não financeiras de capital aberto no período de 2011 a 2015. A pesquisa mostrou que diante do fato dos investidores receberem, diariamente, um grande fluxo de informações e, muitas vezes, não conseguirem lidar com as mesmas (racionalidade limitada). O processamento dessa informação pelo indivíduo se torna impossível, chegando a um nível em que o detalhamento de informações não garante retorno mais elevado para a organização. Esse resultado alinha-se com teorias que afirmam que o excesso de informações pode resultar em uma sobrecarga na análise feita pelas pessoas, fato esse que diminui o comprometimento dos agentes no momento de escolher determinada opção ou mesmo analisar todas as informações disponíveis, gerando sobrecarga no processo de tomada de decisões.

As variáveis relacionadas ao disclosure e à governança corporativa não apresentaram relação estatisticamente significante com as variáveis de valor das companhias. Entretanto, um importante resultado encontrado no presente estudo consiste na reflexão sobre até que ponto a informação é 
necessária para o investidor e quando a mesma extrapola seus limites de compreensão. Na observação dos níveis de transparência, o fator de risco é o primeiro deles, o qual é uma informação simples, rápida, curta e disponível no site da organização. O segundo é a disponibilidade do relatório anual, uma informação mais trabalhosa de se elaborar e organizar, sendo mais difícil para a análise do investidor. O terceiro nível é a qualidade dessas informações, mensurado nesse trabalho pelo RCVP.

O resultado alcançado sugere que os níveis de informação impactam o risco da empresa e não o valor da mesma. Outra informação interessante é o fato de que o nível dessa informação também impacta a percepção do risco, tanto assim que, para o mais elevado RCVP, não foi encontrado significância com nenhuma das regressões em que o mesmo foi testado.

Vale a pena ressaltar que esses resultados contribuem para entender o panorama de disclosure dentro do Brasil, pois mesmo os resultados internacionais aprecem como controversos ou inconclusivos; compreendendo assim a realidade do Brasil, pode-se contribuir para a teoria frente às realidades locais. Já para a prática, o artigo contribui para a decisão de gestores sobre a divulgação voluntária na comunicação empresarial, lembrando que toda dedicação para realização de algo dentro de uma organização gera custo de oportunidade, e tomar decisão bom base em dados é uma das funções do gestor.

Os limites da pesquisa são: geográfico (foram realizadas inferências apenas sobre a realidade brasileira), temporal (foco no período de 2011 a 2015) e metodológico/amostragem (extração dos dados de um dos relatórios dentre os diversos relatórios das empresas, com um recorte do universo das empresas de capital aberto com maior liquidez da bolsa).

Diante dos resultados encontrados e das limitações do artigo, sugerese que em pesquisas futuras seja feito um aprofundamento ainda maior nos níveis de transparência, de modo que outras variáveis relacionadas à mesma sejam utilizadas, como também outros índices sejam desenvolvidos. Sugerese, ainda, que sejam testadas também variáveis relacionadas ao 
desempenho, tais como a margem Líquida, o retorno sobre o ativo e o lucro operacional para que seja estudada a possível relação dessas variáveis com o disclosure.

\section{Referências}

Almeida, M. A. \& Santos, J. F. (2016). Estrutura de capital e divulgação voluntária de informações de responsabilidade social corporativa das empresas brasileira. Revista de Ciências da Administração, 18(45), 109-126. https://doi.org/10.5007/2175-8077.2016v18n45p109

Alves, L. C., Gonçalves, F. V., \& Peixoto, F. M. (2014). Risco e transparência no Brasil: um estudo sobre o gerenciamento de resultados. Revista Brasileira de Administração Científica, 5(3), 203-221. https://doi.org/10.6008/SPC2179-684X.2014.003.0012

Baum, C. F. (2001). Residual diagnostics for cross-section time series regression models. Stata Journal, 1(1), 101-104. https://doi.org/10.1177/1536867X0100100108

Bertomeu, J., Beyer, A., \& Dye, R. A. (2011). Capital structure, cost of capital, and voluntary disclosures. The Accounting Review, 86(3), 857-886. https://doi.org/10.2308/accr.00000037

BM\&FBovespa. Bolsa De Valores, Mercadorias e Futuros de São Paulo. Diretrizes de governança corporativa. Recuperado de http://ri.bmfbovespa.com.br/fck_temp/26_2/Diretrizes_de_Governanca_Corpo rativa_da_BMFBOVESPA.pdf.

Bushman, R. M. \& Smith, A. J. (2003). Transparency, financial accounting information, and corporate governance. Economic Policy Review, 9(1), 65-87.

Carvalhal-da-Silva, A. L. \& Leal, R. P. C. (2005). Corporate Governance Index, firm valuation and performance in Brazil. Revista Brasileira de Finanças, 3(1), 1-18.

Castro Junior, F. H. F., Conceição, P. M., \& Santos, D. (2011). A relação entre o nível voluntário de transparência e o custo de capital próprio das empresas brasileiras não financeiras, Revista Eletrônica de Administração, 70(3), 617-635. https://doi.org/10.1590/S1413-23112011000300002

Chung, K. H. \& Pruitt, S. W. (1994) A simple approximation of Tobin's Q. Financial Management, 23(3), 70-74. https://doi.org/10.2307/3665623

Damodaran, A. (2002). Finanças corporativas aplicadas. Porto Alegre: 
Bookman.

Dantas, J. A., Zendersky, H. C., Santos, S. C., \& Niyama, J. K. (2005). A dualidade entre os benefícios do disclosure e a relutância das organizações em aumentar o grau de evidenciação. Economia e Gestão, 5(11), 56-76.

Doná, A. L., Marques, K. C. M., Moribe, A. M., \& Hercos Junior, J. B.(2015). Fatores determinantes do conteúdo divulgado no relatório de administração de empresas brasileiras. Revista Universo Contábil, 11(4), 82-106. https://doi.org/10.4270/ruc.2015433

Gil, A. C. (2002). Como Elaborar Projetos de Pesquisa. São Paulo: Atlas.

Greene, W. H.(2008). Econometric Analysis. Upper Saddle River: Prentice Hall.

Guay, W. R. \& Verrecchia, R. E. (2018). Conservative disclosure. Journal of Financial Reporting, 3(1), 73-92. https://doi.org/10.2308/jfir-52051

Hausman, J. A. (1978). Specification tests in econometrics. Journal Of The Econometric Society, 46(6), 1251-1271. https://doi.org/10.2307/1913827

Holtz, L. \& Sarlo Neto, A. (2014). Efeitos das características do conselho de administração sobre a qualidade da informação contábil no Brasil. Revista Contabilidade \& Finanças, 25(66), 255-266. https://doi.org/10.1590/1808$\underline{057 x 201412010}$

Instituto Brasileiro de Governança Corporativa (2015). Código das melhores práticas de governança corporativa. Recuperado de http://www.ibgc.org.br/userfiles/2014/files/CMPGPT.pdf.

Iyengar, S. \& Lepper, M. (2000). When Choice is Demotivating: Can One Desire Too Much of a Good Thing? Journal of Personality and Social Psychology, 76(6), 995-1006. https://doi.org/10.1037/0022-3514.79.6.995

Jensen, M. C. \& Meckling, William H.(1976). Theory of the Firm: Managerial Behavior, Agency Costs, and Capital Structure. Journal of Financial Economics, 3(4), 305-360. https://doi.org/10.1016/0304405X(76)90026-X

Kennedy, P. (2009). Manual de Econometria. Rio de Janeiro: Campus Elsevier.

Lameira, V. J.(2012). As Relações entre Governança e Risco nas Companhias Abertas Brasileiras. Revista Brasileira de Gestão de Negócios, 14(42), 7-25. 
Langer, E. J. \& Rodin, J. (1976). The Effects of Choice and Enhanced Personal Responsibility for the Aged: A Field Experiment in an Institutional Setting. Journal of Personality and Social Psychology, 34(2), 191-198. https://doi.org/10.1037/0022-3514.34.2.191

Lanzana, A. P., Silveira, A. D. M., \& Famá, R.(2006, setembro). Existe Relação entre Disclosure e Governança Corporativa no Brasil? Anais do Encontro da Associação Nacional de Pós-Graduação e Pesquisa em Administração, EnANPAD. Salvador, BA. Brasil, 30.

Leuz, C. \& Wysocki, Peter D. (2016). The economics of disclosure and financial reporting regulation: Evidence and suggestions for future research. Journal of Accounting Research, 54(2), 525-622. https://doi.org/10.1111/1475-679X.12115

Lopes, A. B. \& Walker, M. (2008). Firm-Level Incentives and the Informativeness of Accounting Reports: An Experiment in Brazil. Working Paper. Recuperado de http://ssrn.com/abstract=1095781.

Malacrida, M. J. C. \& Yamamoto, M. M. (2006). Governança Corporativa: nível de evidenciação das informações e sua relação com a volatilidade das ações do Ibovespa. Revista Contabilidade \& Finanças. Ed. Comemorativa, 65-79. https://doi.org/10.1590/S1519-70772006000400006

Marques, V. A., Silva, F. G. D., Louzada, L. C., Amaral, H. F., \& Souza, A. A. (2015). Qualidade informacional e nível de transparência: um estudo entre empresas ganhadoras e não ganhadoras do troféu transparência Fipecafi-Serasa Experian. Revista de Administração, Contabilidade e Economia, 14(2), 769-796. https://doi.org/10.18593/race.v14i2.5912

Michelon, G., Pilonato, S. \& Ricceri, F. (2015). CSR reporting practices and the quality of disclosure: An empirical analysis. Critical perspectives on Accounting, 33(C), 59-78. https://doi.org/10.1016/j.cpa.2014.10.003

Moreira, R. L., Colauto, R. D. \& Amaral, H. F. (2010). Conservadorismo condicional: estudo a partir de variáveis econômicas. Revista Contabilidade \& Finanças, 21(54), 64-84. $\quad$ https://doi.org/10.1590/S1519$\underline{70772010000300006}$

Peixoto, F. M. (2012). Governança corporativa, desempenho, valor e risco: estudo das mudanças em momentos de crise (Tese de Doutorado). Universidade Federal de Minas Gerais, Belo Horizonte, MG, Brasil.

Perobelli, F. F. C., \& Ness Jr., W. L. (2000, setembro). Reações do mercado acionário a variações inesperadas nos lucros das empresas: um estudo sobre 
a eficiência informacional no mercado brasileiro. Anais do Encontro da Associação Nacional de Pós-Graduação e Pesquisa em Administração. EnANPAD, Florianópolis, SC, Brasil, 24.

ROCHA, C. F. P.; PROCIANOY, J. L. (2004). Disclosure das companhias abertas brasileiras: um estudo exploratório. GESTÃO.Org - Revista Eletrônica de Gestão Organizacional, 2(3), 1-14.

Schadewitz, H. J. \& Blevins, D. R. (1998). Major Determinants of Interim Disclosures in an emerging market. American Business Review, 16(1), 41-55.

Schulz, R. (1976). Effects of Control and Predictability on the Physical and Psychological Well-being of the Institutionalized Aged. Journal of Personality and Social Psychology, 33(5), 563-573. https://doi.org/10.1037/0022-3514.33.5.563

Silveira, A. D. M. (2004). Governança Corporativa e Estrutura de Propriedade: determinantes e relação com o desempenho das empresas no Brasil (Tese de Doutorado), Universidade de São Paulo, São Paulo, SP, Brasil.

Skaife, H. A., Collins, D. W., \& Lafond, R. (2004). Corporate Governance and the Cost of Equity Capital. Working Paper. Recuperado de SSRN de http://ssrn.com/abstract=639681.

Wong, S. C. Y. (2009).Uses and Limits of Conventional Corporate Governance Instruments: Analysis and Guidance for Reform (Integrated version). Recuperado de Private Sector Opinion, Global Corporate Governance Forum de http://ssrn.com/abstract=1409370.

Wooldridge, J. M. (2002). Econometric Analysis of Cross Section and Panel Data. London: MIT Press. 